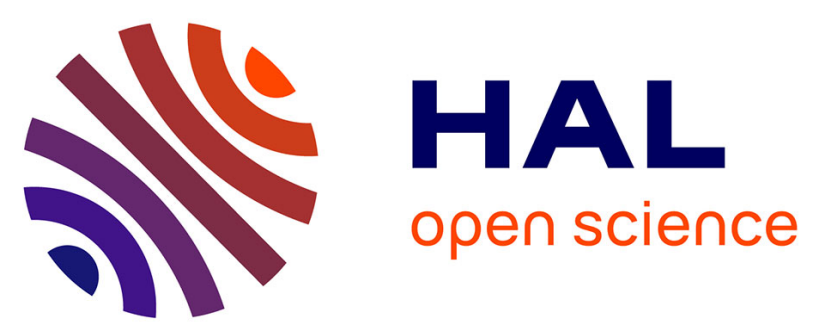

\title{
BENEFIT OF NEURAL NETWORK FOR THE OPTIMIZATION OF DEFECT DETECTION ON COMPOSITE MATERIAL USING ULTRASONIC NON DESTRUCTIVE TESTING
}

Pauline Trouvé-Peloux, Baptiste Abeloos, Achraf Ben Fekih, Camille Trottier, Jean-Michel Roche

\section{To cite this version:}

Pauline Trouvé-Peloux, Baptiste Abeloos, Achraf Ben Fekih, Camille Trottier, Jean-Michel Roche. BENEFIT OF NEURAL NETWORK FOR THE OPTIMIZATION OF DEFECT DETECTION ON COMPOSITE MATERIAL USING ULTRASONIC NON DESTRUCTIVE TESTING. 48th Annual Review of Progress in Quantitative Nondestructive Evaluation (QNDE 2021), Jul 2021, VIRTUEL, United States. hal-03446054

\author{
HAL Id: hal-03446054 \\ https://hal.science/hal-03446054
}

Submitted on 24 Nov 2021

HAL is a multi-disciplinary open access archive for the deposit and dissemination of scientific research documents, whether they are published or not. The documents may come from teaching and research institutions in France or abroad, or from public or private research centers.
L'archive ouverte pluridisciplinaire HAL, est destinée au dépôt et à la diffusion de documents scientifiques de niveau recherche, publiés ou non, émanant des établissements d'enseignement et de recherche français ou étrangers, des laboratoires publics ou privés. 


\section{BENEFIT OF NEURAL NETWORK FOR THE OPTIMIZATION OF DEFECT DETECTION ON COMPOSITE MATERIAL USING ULTRASONIC NON DESTRUCTIVE TESTING}

\author{
P. Trouvé-Peloux; B. Abeloos \\ DTIS, ONERA - Université Paris-Saclay \\ F-91123, Palaiseau, France \\ Email: pauline.trouve@onera.fr
}

\author{
A. Ben Fekih, C. Trottier, J.-M. Roche \\ DMAS, ONERA - Université Paris-Saclay \\ F-92322 Châtillon, France
}

\begin{abstract}
This paper is dedicated to out-of-plane waviness defect detection within composite materials by ultrasonic testing. We present here an in-house experimental database of ultrasonic data built on composite pieces with/without elaborated defects. Using this dataset, we have developed several defect detection methods using the C-scan representation, where the defect is clearly observable. We compare here the defect detection performance of unsupervised, classical machine learning methods and deep learning approaches. In particular, we have investigated the use of semantic segmentation networks that provides a classification of the data at the "pixel level", hence at each $C$-scan measure. This technique is used to classify if a defect is detected, and to produce a precise localization of the defect within the material. The results we obtained with the various detection methods are compared, and we discuss the drawbacks and advantages of each method.
\end{abstract}

\section{INTRODUCTION}

For several years, many computer vision applications such as object detection, scene understanding or 3D vision, have benefited from the huge interest of the data processing community for neural networks approaches. Yet, the benefit of such approaches for the optimization of defect detection in materials and struc-

\footnotetext{
* Address all correspondence to this author.
}

tures using non destructive testing (NDT) is still to be investigated. In this paper, we consider the inspection of composite materials by ultrasonic testing (UT) for out-of-plane waviness defect. Figure 1 shows an illustration of such defect within the material. As simulation of this defect is challenging, we have built an experimental set of composite materials, with and without defect, which have been tested by UT. We study here the benefit of the use of neural network for defect detection on this dataset, compared to unsupervised and more classical machine learning approaches.

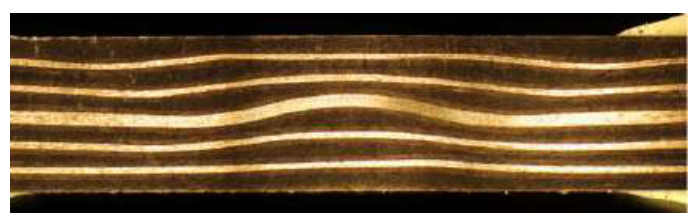

FIGURE 1. ILLUSTRATION OF OUT-OF-PLANE WAVINESS DEFECT ON A COMPOSITE MATERIAL.

\subsection{State of the art}

Among non destructive testing techniques, UT is simple to use with a large variety of materials, including composite and metallic. This method is fast and can provide a precise locali- 
sation of defect within the material. Automation of the testing can generate a large amount of data to be processed. Hence machine learning and deep learning methods have been developed to conduct defect detection either on composite [1-3] or metallic materials [4-9]. One challenge is then to label the data before the learning stage of those methods.

A common approach for defect detection using learning approaches consists in the transformation of the 1D signal (A-scan) into features vector with lower dimension, using for instance wavelet transform [3,6], chirplet transform [2] or directly several convolutional layers of a neural network $[7,8]$. This features vector is then used at the input of a classifier. However, with a defect detection on A-scans, the defect localisation within the material depth is usually lost. An exception is the work of D'Orazio and al. [1] who trained a perceptron to predict the defect localization within three zones of the material : top, middle and bottom. Besides, with defect detecion on A-scan the spatial information is not exploited. To benefit from spatial measure variation, B-scans have also been used in the literature for defect detection $[4,5,9]$. For example, Medak et al. apply an object detection network for a localized defect detection on metallic material with a network trained on a large in-house dataset.

\subsection{Contributions}

In this paper, we build an in-house experimental database of UT data on composite material with/without out-of-plane waviness defect. In contrast with the literature, we propose to detect defects on C-scans in order to benefit from the spatial information contained in each measure surrounding area, and to produce a defect detection within the material depth. We propose here a semantic segmentation of each measures of the C-scan, followed by a threshold on the number of measured classified as with defect. Hence, we obtain a classification of the whole C-scan : with or without defect. The defect detection performance of this approach is compared to other unsupervised and machine/deep learning methods.

\section{EXPERIMENTAL DATABASE}

In this section we describe the elaboration of our set of composite pieces with and without defect. We also describe the experimental set-up for UT used to obtain our database.

\subsection{Material elaboration}

Our composite material pieces are made of the TENCATE CETEX®TC1225 composed of a thermoplastic PAEK matrix and unidirectional fibers in carbon T700GC with a ply thickness of $0.21 \mathrm{~mm}$. To elaborate the pieces without defect, we cut and stacked $233 \times 126 \mathrm{~mm}$ flat plate of either 6,8 or 16 plies. The resulting stack was then put into a mould and placed into a 40 tons press within two heating plates. A heating and pressuring process was finally conducted according to a specific cycle for consolidation of the material.

To generate the out-of-plane waviness defects, we introduced strip within the material plies using three different methods. In the first two methods, a transverse strip was introduced either in the middle or in the bottom of the plies stack. Here, these methods are respectively referred to as M1 and M2. The strip had various width ( $2 \mathrm{~mm}$ or $10 \mathrm{~mm}$ ) and various fiber orientations : $0^{\circ}$ or $90^{\circ}$. Figure 2 top shows an example of a strip placed in the middle of the stack. A third method, referred to as $\mathrm{M} 3$, consisted in the introduction of plies between two discontinuous plies of equal thickness, as illustrated at the bottom of Figure 2. As for the pieces without defect, once the plies stack was elaborated, it was placed into a mold and a specific procedure for heating and pressuring was applied for material consolidation. Finally we obtained a set of 7 pieces without defect, and 8 pieces with defect (5 M1, 2 M2 and $1 \mathrm{M} 3$ ).

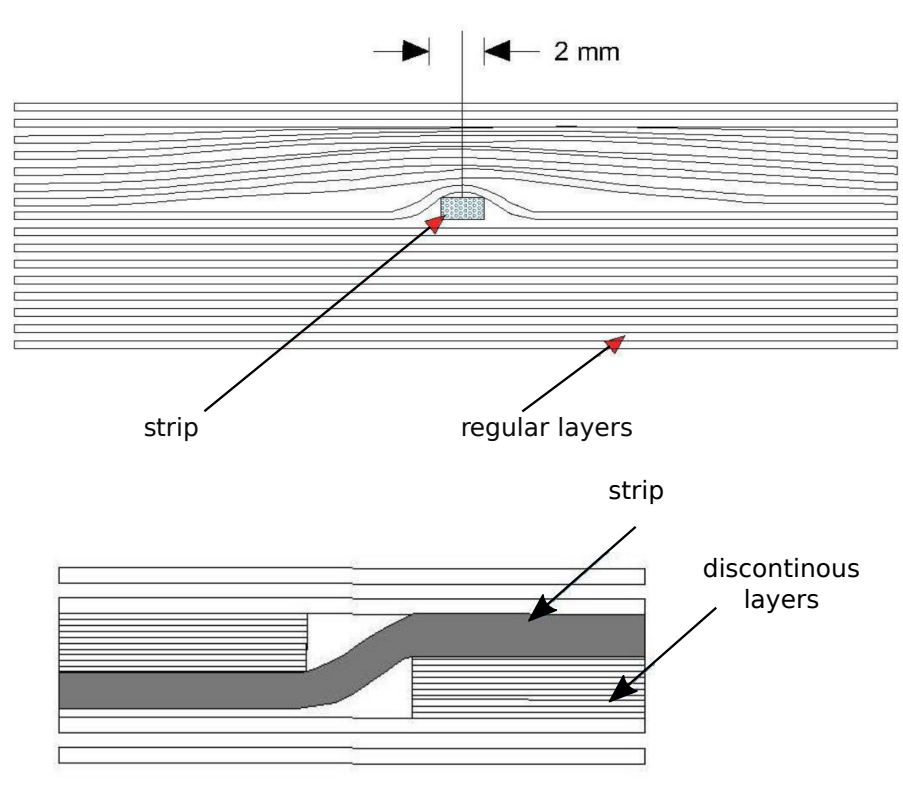

FIGURE 2. EXAMPLES OF OUT-OF-PLANE WAVINESS DEFECT ELABORATION METHODS. TOP : STRIP IN THE MIDDLE OF THE MATERIAL (M1). BOTTOM : STRIP WITHIN A LAYERS DISCONTINUITY (M3).

\subsection{Ultrasonic testing}

For each piece of material, we have conducted UT using the set-up illustrated in Figure 3. The focalized transducer working at $20 \mathrm{MHz}$ is put in total immersion into water. For each piece of composite, 400x400 A-scans were measured with a spatial step 


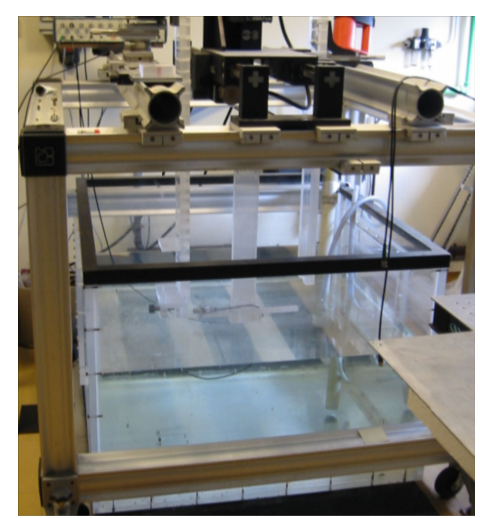

FIGURE 3. ULTRASONIC TESTING SET-UP IN TOTAL IMMERSION AT ONERA.

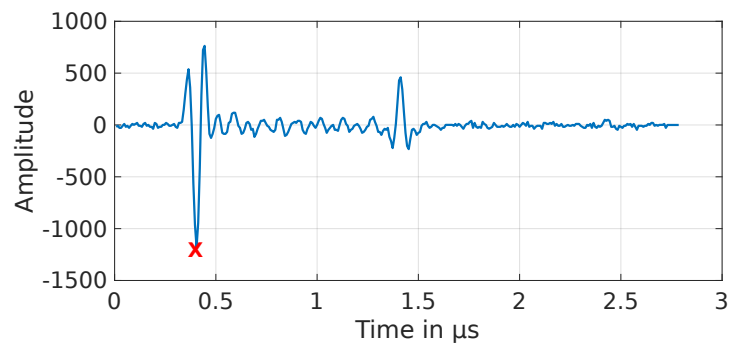

FIGURE 4. EXAMPLE OF A-SCAN. THE RED CROSS CORRESPONDS TO THE SURFACE REFERENCE FOR TEMPORAL REALIGNMENT.

of $0.5 \mathrm{~mm}$. Figure 4 shows an example of A-scan acquired with our system.

\section{DATA PRE-PROCESSING AND DATABASE}

Before application of a processing for defect detection, we used few pre-processing steps that are described in the following.

\subsection{Temporal realignment of the A-scans}

The piece of material are slightly tilted as illustrated in Figure 5 left. To correct this tilt, we realigned the A-scans according to the same surface reference as illustrated with the red cross in Figure 4. Thus, for a material without defect, all the signals at a given time corresponds to the same material layer. This processing made the defect more clearly observable as illustrated in the B-scan and C-scan in Figures 5 and 6.

After the temporal alignment, we observed that defect appeared more clearly on the $\mathrm{C}$-scans than on the B-scans. Thus, we have chosen to work with this representation to conduct the defect detection. Moreover, this representation is more comparable to an image, which opens the path to the use of computer vision methods such as semantic segmentation. In our case, each
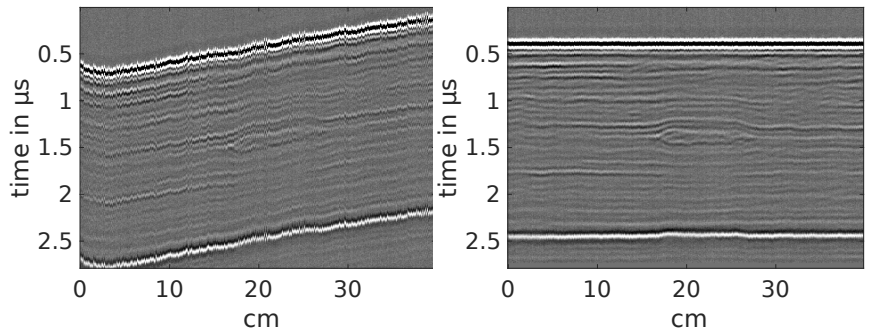

FIGURE 5. ILLUSTRATION OF TEMPORAL REALIGNMENT EFFECT ON THE B-SCAN: LEFT - ORIGINAL B-SCAN, RIGHT REALIGNED B-SCAN.
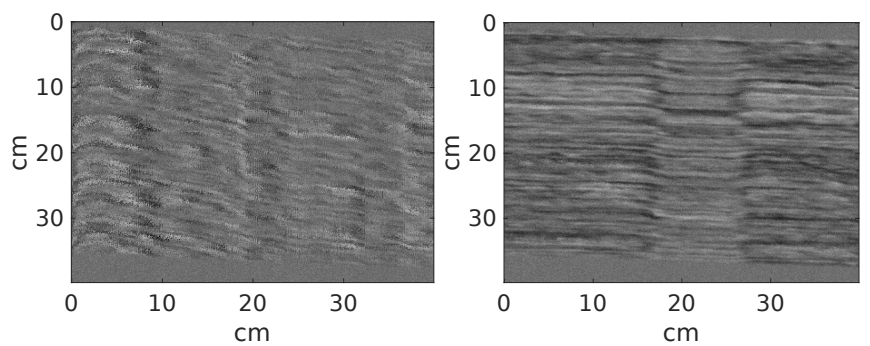

FIGURE 6. ILLUSTRATION OF TEMPORAL REALIGNMENT EFFECT ON THE B-SCAN: LEFT - ORIGINAL C-SCAN, RIGHT REALIGNED C-SCAN.

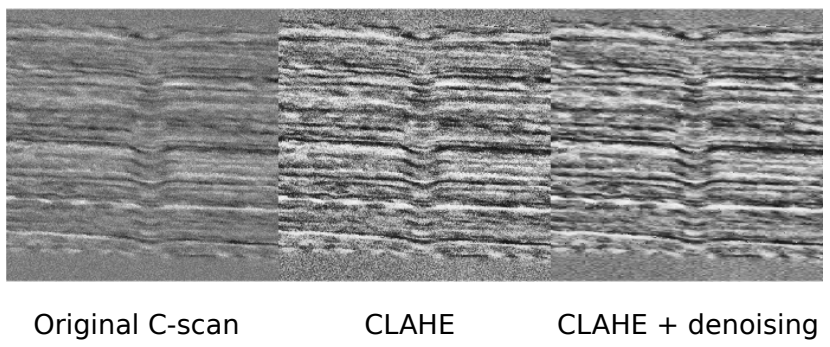

FIGURE 7. CONTRAST ENHANCEMENT PRE-PROCESSING.

original C-scan was of size $400 \times 400$ measures. Note that in the following, we will refer to each value of the $\mathrm{C}$-scan with the term pixel as for images.

\subsection{Contrast enhancement and rotation correction}

After the temporal realignment, we used common processing for contrast enhancement such as CLAHE method, followed by a denoising using a truncation within the data wavelength decomposition. Finally, since we observed a small rotation of the pieces with respect to $\mathrm{XY}$ axis, we estimated the angle and rotated the C-scans accordingly so that they have all the same orientation. Figures 7 and 8 illustrate the effect of the contrast enhancement and the rotation correction. 


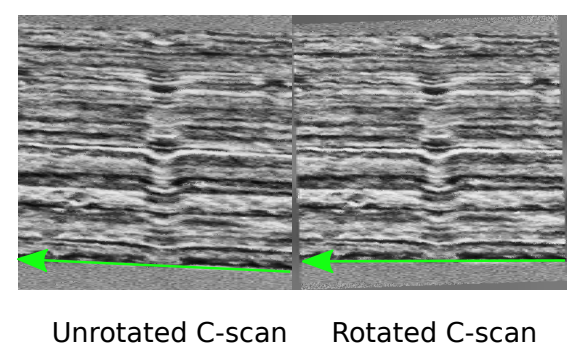

FIGURE 8. ROTATION CORRECTION OF THE C-SCAN.

\subsection{Database}

Given our in-house pieces of composite material, we separated the $\mathrm{C}$-scans with and without defect into training and testing database. For each piece of material, we removed the $\mathrm{C}$-scan corresponding to the very first and last surfaces, where no defect was observable. Since the defect did not affect all the layers of the material, we manually selected the $\mathrm{C}$-scan in which the defect was visible to build the dataset with defect. Hence, we obtained $1494 \mathrm{C}$-scan extracted from 6 pieces of material with defect, and $1312 \mathrm{C}$-scan without defect extracted from 5 pieces of material. For testing, we used $412 \mathrm{C}$-scans with defect extracted from two pieces of material and $531 \mathrm{C}$-scan without defect extracted from two pieces of material. Figure 9 shows example of C-scans of the training and testing database.
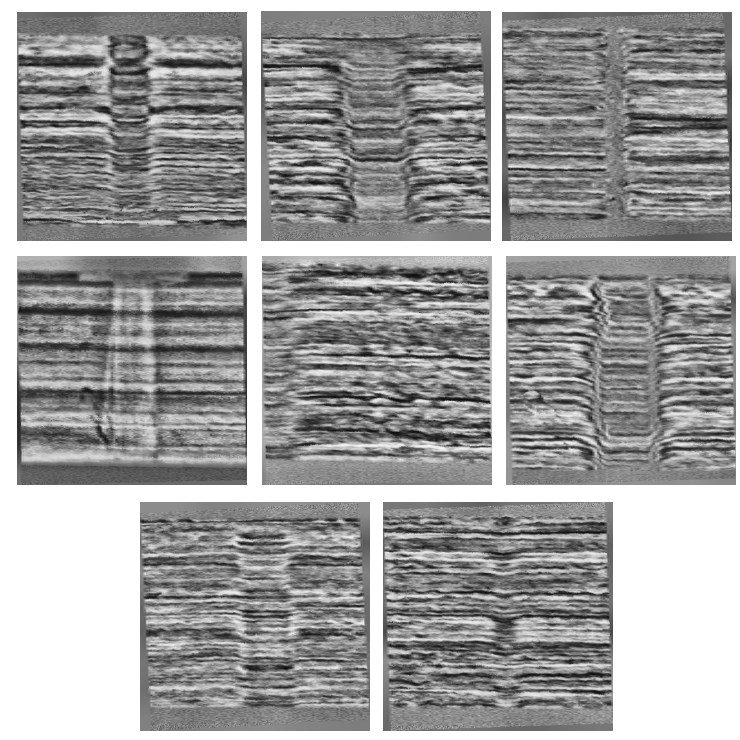

FIGURE 9. ILLUSTRATION OF C-SCANS FROM THE TRAINING (FIRST TWO ROWS) AND TESTING (LAST ROW) DATABASES.

Note that in the examples provided in Figure 9, the defects appear obviously but the database also contained C-scans with
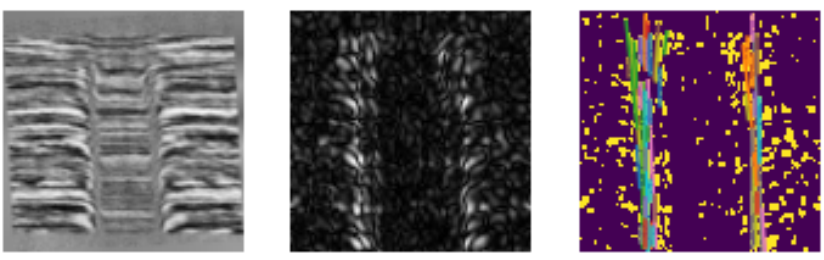

FIGURE 10. EXAMPLE OF RESULT OF THE HOUGH SEGMENT DETECTION METHOD. FROM LEFT TO RIGHT : ORIGINAL C-SCAN, RESULT OF VERTICAL SOBEL FILTER AND HOUGH SEGMENT DETECTION RESULT.

less visible defects as shown in Figure 18.

\section{PERFORMANCE METRICS}

To evaluate the performance of the defect classification problem on C-scans, we used the following performance metrics: precision, recall and $\mathrm{F} 1$ score. Such metrics are defined using the rate of true positive $(T P)$, false negative $(F N)$ and false positive $(F P)$ :

$$
\begin{aligned}
\text { Precision } & =\frac{T P}{T P+F P} \quad F 1=2 \frac{\text { Precision } \times \text { Recall }}{\text { Precision }+ \text { Recall }} \\
\text { Recall } & =\frac{T P}{F N+T P} \quad \text {. }
\end{aligned}
$$

\section{UNSUPERVISED DETECTION METHOD}

The visual aspect of the defect within the C-scan, firstly led us to develop an unsupervised method based on segment detection. To do so, we applied a Sobel filter on subsampled C-scan of size $128 \times 128$ in order to emphasis the gradient in the vertical direction. Then, we binarised the result before applying a Hough detection algorithm [10]. The C-scan was considered as with defect if more than one segment was detected. Figure 10 illustrates the result of the Hough segment detection and quantitative detection scores are given in Table 3 . We obtain a precision of 1 , which means that there is no false positive. However the recall is low, meaning that there is a high number of false negative. This can be understandable looking for instance on the second $\mathrm{C}$-scan example in the test shown in Figure 9. There is no obvious segment that can be seen in the image while there is actually a defect.

This unsupervised method is fast and does not require a large database. However, the binarisation and the Hough detection method rely on several thresholds. Here, we manually fixed them in order to get satisfactory detection results. Besides, it is based on the assumption of a vertical defect, which is the case in our database, but it won't be necessary true for a real defect. 

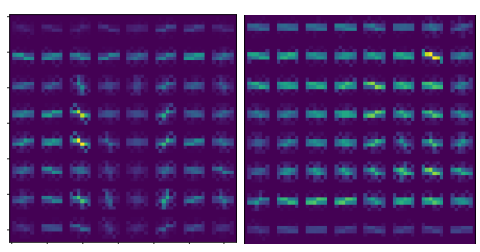

FIGURE 11. APPLICATION OF HOG DESCRIPTORS ON THE CSCAN. LEFT : WITH DEFECT. RIGHT: WITHOUT DEFECT.

\section{MACHINE LEARNING APPROACH}

The support vector machine classifier (SVM) [11] is a machine learning approach that finds the best hyper-plan that separates the data. The plan is obtained by maximizing the margin between the closest data with respect to this plan. This technique does not require a large dataset to provide satisfactory results when the data is linearly separable. The SVM method leverages on the choice of features at the input of the SVM. We tested common features that are the histogram of Gradient (HOG) [12] and the Gabor filters [13]. To reduce inputs dimension, these filters have been applied on a scaled version of the $\mathrm{C}$-scans of size $64 \times 64$ pixels. Figure 6 shows the result of the HOG descriptors applied on a C-scan with defect (left) and without defect (right). The orientation of the gradients clearly changes along the defect position. Table 3 shows the performance results of this method for both descriptors. HOG descriptors obtained here the best results with in particular a higher recall score than the segment detection method.

\section{DEEP LEARNING APPROACHES}

\subsection{Classification network}

Previous method based on SVM requires the definition of features for descriptors extraction that reduces the input dimension. Deep learning methods avoid such definition and the features are directly learned during the training. However this requires a longer training and a large database. We used a simple classification network made of few convolutional layers followed by two fully connected layers and a softmax classifier, as illustrated in Figure 12. In order to overcome the small size of the dataset, the parameters of the network have been pre-trained on CIFAR10 dataset ${ }^{1}$. Hence the C-scans are scaled to the size $32 \times 32$, as the images of this dataset. We also conducted data augmentation using horizontal translation. Table 3 presents the results obtained with this method. The classification network outperforms the unsupervised and the SVM approaches with a F1 score above 0.91. Yet during the training we observed some instabilities with convergence to local aberrant minimima. We believe that this effect could be solved with more data.

\footnotetext{
${ }^{1}$ https://www.cs.toronto.edu/ kriz/cifar.html
}

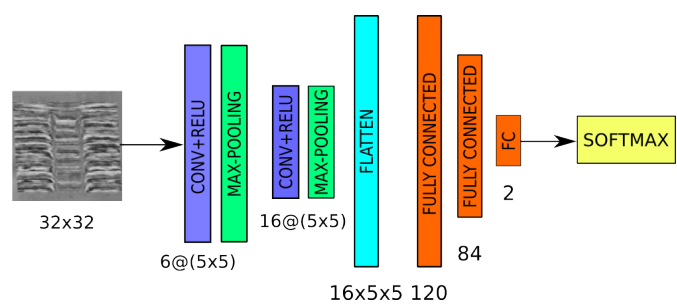

FIGURE 12. CLASSIFICATION NETWORK ARCHITECTURE.

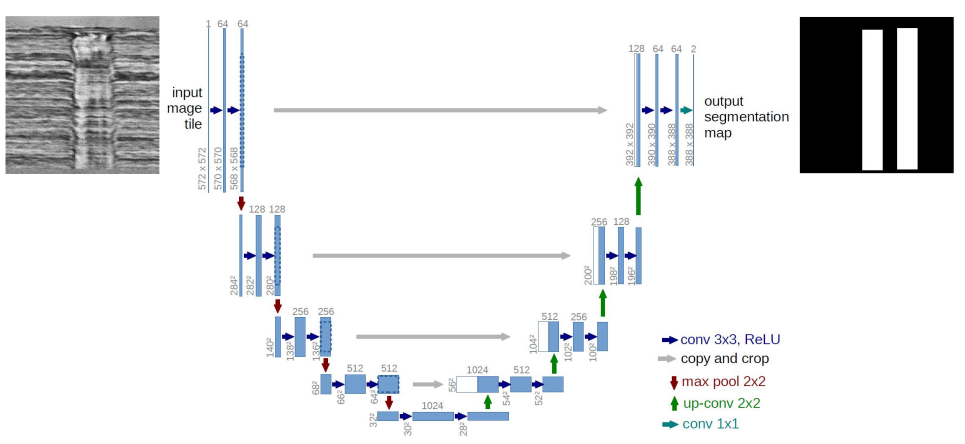

FIGURE 13. U-NET ARCHITECTURE FOR C-SCAN SEMANTIC SEGMENTATION. [14]

\subsection{Semantic segmentation and threshold classifica- tion}

Principle: The objective of this approach is to classify each pixel between two categories: defect or without defect, and then to classify the $\mathrm{C}$-scan by applying a threshold on the ratio of the detected defect pixels. The semantic segmentation task is based on the training of deep convolutional neural networks such as U-Net [14] as illustrated in Figure 13. The network takes the images of C-scans as input, and is trained to detect each defect pixel. The first part of the network is the contracting path, or encoder that enables to capture the context, while the second part of the network is a symmetric expanding path, or decoder that enables the image reconstruction and localization. Each side of the defect have been previously manually labelled as illustrated in the right side of Figure 13. The network is trained by minimizing the cross entropy between the segmentation and the labels. Once each pixel have been classified, a threshold is applied on the defect pixel ratio to classify the entire image as corresponding to a defect or not.

This method enables to precisely localize the defect. It can also be considered as more robust since we have more labelled data at the pixel level than at the image level. However, the major drawback is the need of a labelled dataset which can be time consuming to produce and costly.

Experimentation: Several architectures have been trained and compared: U-Net [14], SegNet [15], and SegNet- 


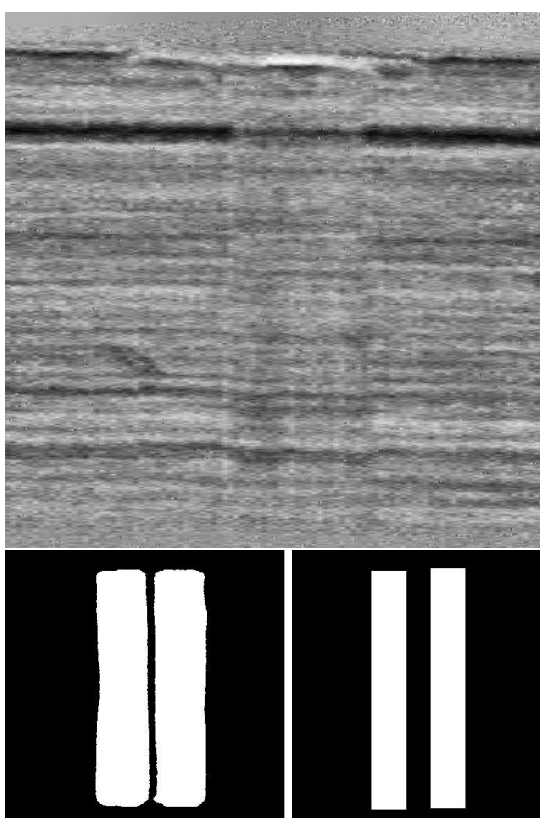

FIGURE 14. ILLUSTRATION OF A DEFECT (UP), THE U-NET SEGMENTATION (BOTTOM LEFT), AND THE ASSOCIATED LABEL (BOTTOM RIGHT).

bn-relu [16]. For each training, we applied a grid search optimisation strategy, varying the learning rates between: [0.01, $0.05,0.001,0.005]$, and the batch size between: [0.01, 0.05, $0.001,0.005]$. The model with the best F1 score have been selected. Before the training, we initialized the network weights using pre-trained models publicly available [16]. For a better generalization, that data have been augmented using random crop technique, and horizontal and vertical flips.

Results: The results of the semantic segmentation task are presented in Table 1. The table presents the F1 scores, precision, and recall for each of the tested architecture. F1 score are very similar and reaches 0.83 with the SegNet-bn-relu. The best precision is obtained with SegNet $(88 \%)$, while the best recall is obtained with U-Net and SegNet-bn-relu (79\%). The lower value of the recall can be explained by the fact that the models tend to partially miss some defect regions due to hardly visible defects, while false positive defects are very rare.

The classification performance based on the threshold of defect pixel ratio is presented in Table 2. A large threshold tends to provide a very good precision and decrease the recall, while a small threshold tends to provide a good recall with a poor precision. We optimised the threshold using the F1 score, giving a very similar contribution to precision and recall. We fixed the threshold at 3\%, and obtained a F1 score for the image classification of $99.6 \%$, and a precision and recall of respectively $100 \%$

\begin{tabular}{|c|c|c|c|}
\hline Method & F1 & Precision & Recall \\
\hline U-Net [14] & 0.82 & 0.86 & $\mathbf{0 . 7 9}$ \\
\hline SegNet [15] & 0.82 & $\mathbf{0 . 8 8}$ & 0.77 \\
\hline SegNet-bn-relu [16] & $\mathbf{0 . 8 3}$ & 0.87 & $\mathbf{0 . 7 9}$ \\
\hline
\end{tabular}

TABLE 1. DETECTION PERFORMANCE OF SEVERAL SEMANTIC SEGMENTATION NETWORKS.

\begin{tabular}{|c|c|c|c|}
\hline Threshold & F1 & Precision & Recall \\
\hline $10 \%$ & 0.989 & 1.0 & 0.978 \\
\hline $\mathbf{3 \%}$ & $\mathbf{0 . 9 9 6}$ & $\mathbf{1 . 0}$ & $\mathbf{0 . 9 9 3}$ \\
\hline $2 \%$ & 0.988 & 0.983 & 0.993 \\
\hline
\end{tabular}

TABLE 2. CLASSIFICATION PERFORMANCE USING DEFECT PIXEL RATIO THRESHOLD (U-NET).

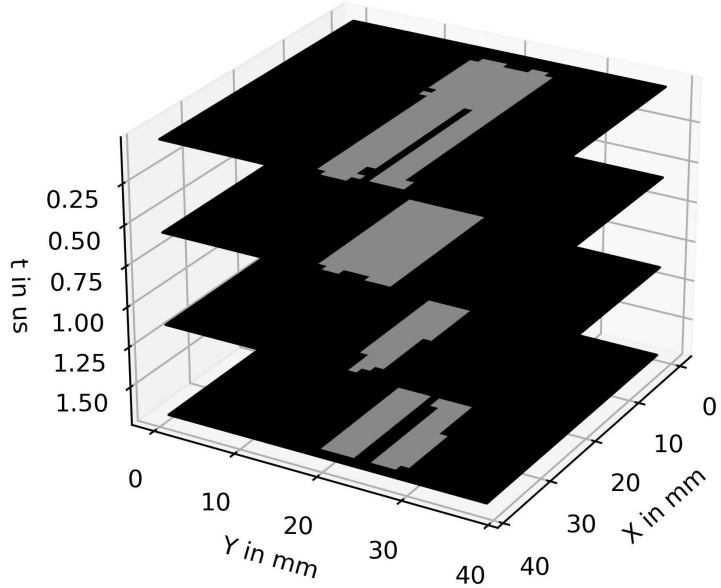

FIGURE 15. ILLUSTRATION OF A CUT PLAN.

and $99.3 \%$. For a better generalization, a larger dataset with more defect diversity would be needed.

The C-scan segmentation can be used to generate a cut plan of each piece as illustrated in Figure 15, while a full 3D reconstruction is illustrated in Figure 16. The reconstruction can be used to estimate the shape of the defect with respect to its depth. In 16 , we can also see false positive regions closed at the border of the plot. These effects that we called "side effects" are described in the following.

Discussion: We investigated the rare cases where the model didn't behave as expected. Figure 17 illustrates an incomplete detection of the defect on the right side. The defect is hardly visible and the detection is only partial. This case is 


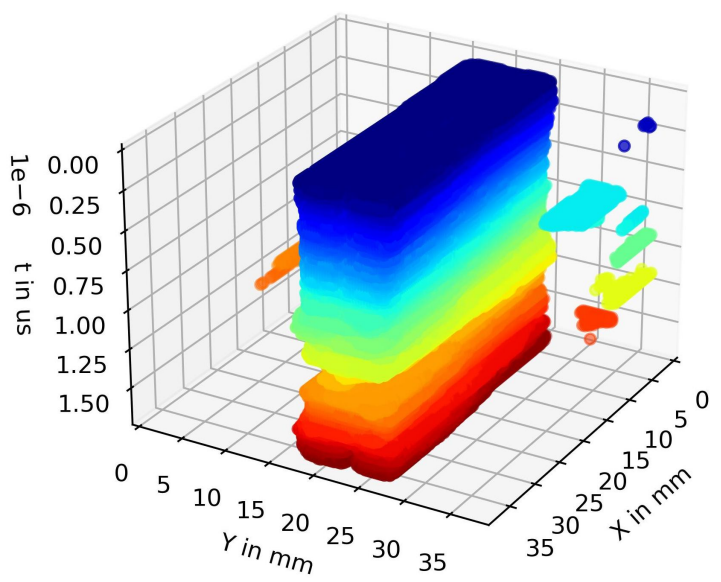

FIGURE 16. ILLUSTRATION OF A 3D RECONSTRUCTION.

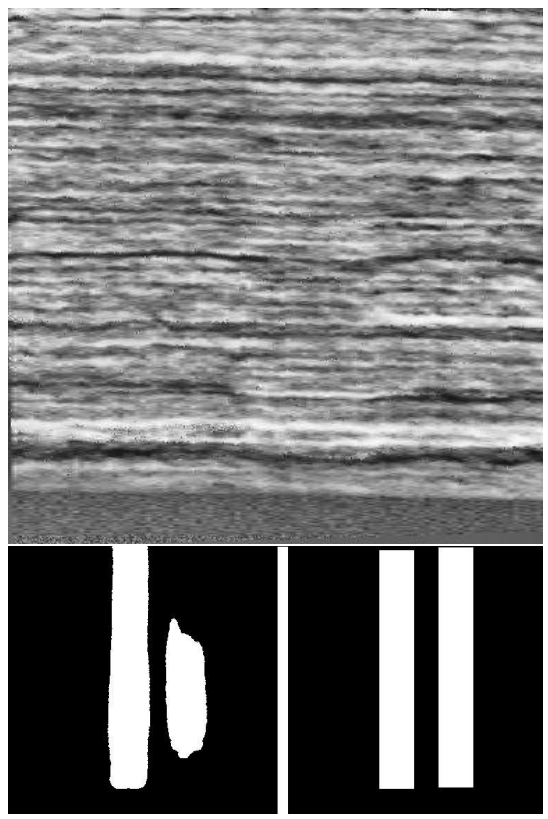

FIGURE 17. PARTIAL DETECTION OF THE DEFECT.

one of the major contributions that decrease the recall. The other main reason is that the labels doesn't fit perfectly the defects, but indicate the region of the defect. Therefore, the model learns to detect the defect and the region around it. A more accurate labelling of the defect would solve this issue, but it is long and no easy to do in practice.

Figure 18 illustrates a rare example where the defect is not detected on the C-scan. As presented in Table 2, this case represents less than $1 \%$ of our data. The reason why the network didn't detect the defect is not clearly understood yet. The interpretability and trustability of the neural networks is one of the major current deep learning challenges. However, the other C-

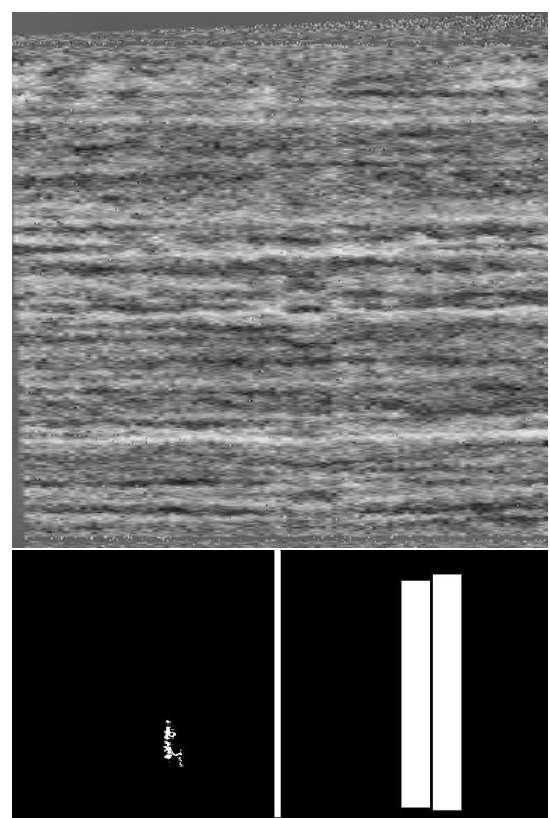

FIGURE 18. MISSING DEFECTION OF THE DEFECT.

scans closed to this depth have been correctly detected as illustrated in the $3 \mathrm{D}$ reconstruction.

A side effect is illustrated in Figure 19. We can see vertical lines in the left part of the figure. These lines have been interpreted as a defect by the model as we can see in the right part. Side effects have probably been caused by the data acquisition processes or the data pre-processing, and could be removed by image truncation.

Table 3 summarizes the results obtained with all the tested methods. The best results measured with the F1 score, precision, and recall are obtained with the semantic segmentation methods + threshold with respectively $99.6 \%, 100 \%$, and $99.3 \%$.

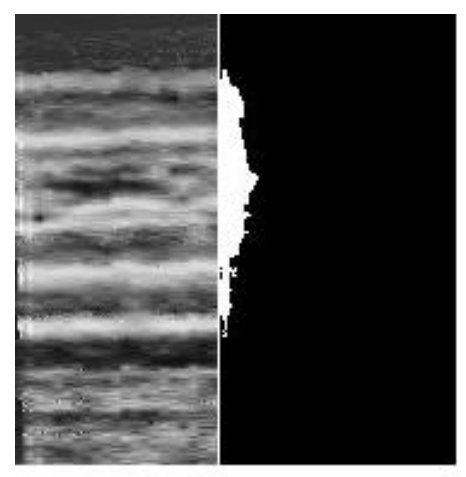

FIGURE 19. ILLUSTRATION OF A SIDE EFFECT. 


\begin{tabular}{|c|c|c|c|}
\hline Method & F1 & Precision & Recall \\
\hline Segment detection & 0.82 & 1 & 0.70 \\
\hline HOG + SVM & 0.86 & 0.96 & 0.77 \\
\hline Gabor filters & 0.80 & 0.91 & 0.71 \\
\hline Classification Network & 0.91 & 0.91 & 0.91 \\
\hline Semantic segmentation + threshold & $\mathbf{0 . 9 9 6}$ & $\mathbf{1 . 0}$ & $\mathbf{0 . 9 9 3}$ \\
\hline
\end{tabular}

TABLE 3. DETECTION PERFORMANCE OF ALL TESTED METHODS.

\section{CONCLUSION}

In this paper, we have presented an experimental ultrasonic database for out-of-plane waviness defect in composite material. The database has been used to develop several defect detection methods based on the $\mathrm{C}$-scan representation where the defects appear clearly. We compared various techniques including unsupervised, supervised and machine/deep learning approaches. The best detection performances have been obtained with the proposed semantic segmentation followed by a threshold for the C-scan classification task. This approach obtained nearly perfect detection score on a C-scans. Moreover the semantic segmentation enables the defect localization within the material. Hence $3 \mathrm{D}$ representation of the defect can be generated.

Perspectives of this work is to improve the data quality in order to avoid sides effect that have been observed in our results, and to obtain more experimental data with larger variety of defects. Improvement of the threshold approach of the semantic map could be obtained using for instance a classification layer to avoid a manual choice of the threshold level. Comparison of defect localization on C-scan could also be compared to B-scan localization such as the one proposed by Medak et al. [9]. Finally, the next step after detection is to characterize the defect, which would be the subject of further studies.

\section{REFERENCES}

[1] D’orazio, T., Leo, M., Distante, A., Guaragnella, C., Pianese, V., and Cavaccini, G., 2008. "Automatic ultrasonic inspection for internal defect detection in composite materials". NDT \& e International, 41(2), pp. 145-154.

[2] Aldrin, J. C., and Forsyth, S., 2012. "Demonstration of using signal feature extraction and deep learning neural networks with ultrasonic data for detecting challenging discontinuities in composite panels". In AIP Conference Proceedings, Vol. 2102, p. 020012.

[3] Meng, M., Chua, Y. J., Wouterson, E., and Ong, C. P. K., 2017. "Ultrasonic signal classification and imaging system for composite materials via deep convolutional neural networks". Neurocomputing, 257, pp. 128-135.
[4] Lawson, S. W., and Parker, G. A., 1996. "Automatic detection of defects in industrial ultrasound images using a neural network". In Vision Systems: Applications, Vol. 2786, International Society for Optics and Photonics, pp. 37-47.

[5] Ye, J., Ito, S., and Toyama, N., 2018. "Computerized ultrasonic imaging inspection: From shallow to deep learning”. Sensors, 18(11).

[6] Virupakshappa, K., Marino, M., and Oruklu, E., 2018. "A multi-resolution convolutional neural network architecture for ultrasonic flaw detection". In 2018 IEEE International Ultrasonics Symposium (IUS), pp. 1-4.

[7] Yan, Y., Liu, D., Gao, B., Tian, G., and Cai, Z., 2020. "A deep learning-based ultrasonic pattern recognition method for inspecting girth weld cracking of gas pipeline". Sensors, 20(14), pp. 7997-8006.

[8] Virkkunen, I., Koskinen, T., Jessen-Juhler, O., and RintaAho, J., 2021. "Augmented ultrasonic data for machine learning". Journal of Nondestructive Evaluation, 40(1), pp. 1-11.

[9] Medak, D., Posilović, L., Subašić, M., Budimir, M., and Lončarić, S., 2021. "Automated defect detection from ultrasonic images using deep learning". IEEE Transactions on Ultrasonics, Ferroelectrics, and Frequency Control, 68(10), pp. 3126-3134.

[10] Guil, N., Villalba, J., and Zapata, E. L., 1995. “A fast hough transform for segment detection". IEEE Transactions on Image Processing, 4(11), pp. 1541-1548.

[11] Hearst, M., Dumais, S., Osuna, E., Platt, J., and Scholkopf, B., 1998. "Support vector machines". IEEE Intelligent Systems and their Applications, 13(4), pp. 18-28.

[12] Dalal, N., and Triggs, B., 2005. "Histograms of oriented gradients for human detection". In 2005 IEEE Conference on Computer Vision and Pattern Recognition, Vol. 1, pp. 886-893 vol. 1.

[13] Fogel, I., and Sagi, D., 1989. "Gabor filters as texture discriminator”. Biological cybernetics, 61(2), pp. 103-113.

[14] Ronneberger, O., Fischer, P., and Brox, T., 2015. "U-net: Convolutional networks for biomedical image segmentation". CoRR, abs/1505.04597.

[15] Badrinarayanan, V., Kendall, A., and Cipolla, R., 2017. "Segnet: A deep convolutional encoder-decoder architecture for image segmentation". IEEE Transactions on Pattern Analysis and Machine Intelligence, 39(12), pp. 24812495.

[16] ONERA, Delta Toolbox 2019. https:/github.com/deltaonera/delta ${ }_{t} b /$ tree/master/deltatb/networks /. 